\title{
Selective Intra-Arterial 177Lu-PSMA Therapy for Castration Resistant Prostate Cancer: Preliminary Results of a Pilot Study
}

\section{Haluk Sayman ( $\nabla$ hbsayman@yahoo.com )}

Istanbul Universitesi-Cerrahpasa Cerrahpasa Tip Fakultesi https://orcid.org/0000-0002-0954-5707

\section{Fatih Gulsen}

Istanbul Universitesi-Cerrahpasa Cerrahpasa Tip Fakultesi

\section{Sait Sager}

Istanbul Universitesi-Cerrahpasa Cerrahpasa Tip Fakultesi

\section{Elife Akgun}

Istanbul Universitesi-Cerrahpasa Cerrahpasa Tip Fakultesi

\section{Nami Yeyin}

Istanbul Universitesi-Cerrahpasa Cerrahpasa Tip Fakultesi

\section{Seckin Bilgic}

Istanbul Universitesi-Cerrahpasa Cerrahpasa Tip Fakultesi

\section{Kubra N. Toplutas}

Istanbul Universitesi-Cerrahpasa Cerrahpasa Tip Fakultesi

\section{Fatih Beytur}

Istanbul Universitesi-Cerrahpasa Cerrahpasa Tip Fakultesi

\section{Rahmi Oklu}

Mayo Clinic Arizona

\section{Omer Aras}

Memorial Sloan Kettering Cancer Center

\section{Original Article}

Keywords: 177Lu-PSMA Therapy, prostate cancer, intra-arterial, castration resistant

Posted Date: February 4th, 2021

DOl: https://doi.org/10.21203/rs.3.rs-173258/v1

License: () (1) This work is licensed under a Creative Commons Attribution 4.0 International License. Read Full License 


\section{Abstract}

Purpose

${ }^{177} \mathrm{Lu}$-PSMA is a promising therapy for patients with metastatic castration resistant prostate cancer patients (mCRPC). To our knowledge, no study has compared the pharmacokinetic profiles of ${ }^{177}$ Lu-PSMA therapy delivered intra-arterially. We aimed to compare the feasibility and safety of selective intra-arterial (IA) administration vs conventional intravenous (IV) administration of ${ }^{177}$ Lu-PSMA in mCRPC.

Methods

In this within-patient pilot study, four patients (median age, 62.5; range, 53-72) with mCRPC who were referred to our clinic between January 2018 and September 2019 for treatment with ${ }^{177}$ Lu-PSMA. Patients were treated in two visits; in each visit receiving half of the total empiric therapeutic dose of $200 \mathrm{mCi}, I \mathrm{Vat}$ the first visit and $I A$ at the second visit. They were followed upto 8 weeks using routine parameters such as hematological status, renal function, and serum PSA levels. For each patient, organ biodistribution in lesions, kidneys, liver, bone marrow, prostate, and whole body was calculated for both administration routes according to the Medical Internal Radiation Dose (MIRD) schema. Mean absorbed doses (MAD) across all patients were compared between both administration routes.

Results

The MAD of all metastatic lesions was significantly higher by $I A$ vs $I V$ administration (1.59 vs 1.20 MGy/MBq; $\mathrm{p}=0.024)$. The prostate gland had a lower MAD between $I A$ and $I V$ administrations, but the difference was not significant ( 0.27 vs $0.36 \mathrm{MGy} / \mathrm{MBq} ; \mathrm{p}=0.22)$. Patients tolerated $I A$ administration very well without unexpected side effects.

Conclusion

These preliminary results of an ongoing study showed that $I A{ }^{177}$ Lu-PSMA therapy is feasible and safe to treat metastatic lesions, potentially having a higher delivery efficacy compared to $I V$ administration.

This study is approved by Cerrahpasa Medical Faculty ethics committee on March 5, 2019 and retrospectively registered with file no: 83045809-604.01.02.

\section{Introduction}

Metastatic castration-resistant prostate cancer (mCRPC) is associated with poor prognosis.

Chemotherapeutic agents, including abiraterone, enzalutamide, and olaparib, show limited survival benefit [1-3]. While overall survival has been reported to improve with radium-223 by 3.6 months, radium-223 is indicated for only patients with skeletal metastases [4]. Additionally, while immunotherapy with sipuleucelT has been reported to confer survival benefit of a few months, it was associated with adverse immunological events and had no effect on time-to-progression [5]. 
Prostate-specific membrane antigen (PSMA) is a glutamate carboxypeptidase II (GCPII), 750-amino-acid type II transmembrane glyco-protein that is overexpressed in prostate cancer [6]. Structurally, it is made up of a 19-amino-acid internal portion, a 24-amino-acid transmembrane portion, and a 707-amino-acid external portion. PSMA expression in malignant tissue is upregulated under androgen deprivation therapy and increases in poorly differentiated, metastatic, and hormone-refractory cancers. These small molecules complexed with Lu-177 penetrate solid tumors and have the advantage of rapid clearance from the blood as compared with whole antibodies, which enables the delivery of high doses of cell destructive beta radiation to tumors whiles limiting adjacent bone marrow irradiation. Lu-177 labeled PSMA radiopharmaceuticals ( ${ }^{177}$ Lu-PSMA) administered intravenously have been shown to bind rapidly to PSMA receptors and become internalized in cells whereby they remain with a half-life of 6.7 days and have a maximal tissue penetration of approximately 2 mm, exerting a crossfire effect (Fig. 1).

The aim of this pilot study was to compare the feasibility and safety of selective intra-arterial $(I A)$ vs conventional intravenous ( $I M$ ) administration of ${ }^{177}$ Lu-PSMA in patients with metastatic castration resistant prostate cancer (mCRPC). A secondary aim was to deliver the highest possible dose in the primary tumor site based on receptor density at first pass, via IA administration, without compromising uptake in any distant soft tissue or bone metastasis.

\section{Materials And Methods}

\section{Patients and Study Design}

The Cerrahpasa medical faculty ethics committee approved this study, and four patients with mCRPC who were referred to our clinic for radionuclide ligand treatment with ${ }^{177}$ Lu-PSMA provided written informed consent after the consenting investigator explained the differences between the two administration routes and study expectations.

In this study to compare two administration routes of ${ }^{177}$ Lu-PSMA, each patient was treated during January 2018-September 2019, in two visits whereby in each visit, each patient received half of the total empiric therapeutic dose of $200 \mathrm{mCi}$. Since prostate cancers are highly heterogeneous cancers, this withinpatient study design had the advantage of automatically correcting for any differences in tumor characteristics between patients. Furthermore, the study design increased statistical power, so that fewer patients had to be treated to determine an effect. The first half of the total dose was administered in the conventional way through an intravenous line. One week after, the second half of the dose was administered selectively using both left and right internal iliac arteries consecutively, through a singlesided femoral catheterization under fluoroscopy. Urethral and capsular arteries are both fed by common prostatic arteries mostly originating from one of four arteries (internal pudendal artery [34.1\%], superior vesical artery [20.1\%], common anterior gluteal-pudendal trunk [17.8\%], and obturator artery [12.6\%]) that are fed by internal iliac arteries. The catheterization of internal iliac arteries was determined to be the most convenient way to administer the radiopharmaceutical for the purpose of this study. Since the study did not involve embolization, there was no need for a super-selective approach. By this means, we were able to 
establish the maximum possible dose rate in the primary tumor site in the first pass. Afterwards, activity was circulated in consecutive cycles to reach other possible metastatic sites within the body.

Patient serum prostate specific antigen (PSA) levels, total blood count, hematocrit, and hemoglobulin levels were obtained, before and every four weeks until two months after the second visit. Toxicity was recorded according to the Common Terminology Criteria for Adverse Events (CTCAE), version 5.0 [7].

\section{Preparation of ${ }^{177}$ Lu-PSMA}

The radiolabeling of PSMA-617 (CMR, Moscow, Russia) was performed in a hot cell using Lu-177 $\mathrm{Cl}_{3}(47$ $\mathrm{MBq} / \mathrm{nmol}$ of ligand) in $0.1 \mathrm{~mol} \mathrm{~L}^{-1} \mathrm{HCl}$ (Eczacıbası Monrol, Turkey) with sodium ascorbate buffer $\mathrm{pH} 4.5$ (Polatom, Otwock-Swierk Poland) at $95^{\circ} \mathrm{C}$ for 20 minutes. After the reaction vial was cooled to room temperature, the volume was adjusted to $2 \mathrm{~mL}$ with saline, and $0.5-1.0 \mathrm{~mL}$ of sterile DTPA solution ( $3 \mathrm{mg}$ $\mathrm{mL}^{-1}$ DTPA in saline) was added. After sterile filtration of this preparation to a sterile vial, the volume was adjusted to $10 \mathrm{~mL}$ with sterile saline under aseptic conditions. Radiochemical purity was determined to be $\geq 98 \%$ by instant thin layer chromatography (ITLC)-silica gel and radio high performance liquid chromatography (HPLC).

\section{Intravenous ${ }^{177}$ Lu-PSMA Administration}

All patients received the first treatment of ${ }^{177}$ Lu-PSMA (first half of the total dose $=100 \mathrm{mCi}$ ) intravenously, with slow infusion using a closed infusion equipment when the patients were in fasting state.

\section{Intra-Arterial ${ }^{177}$ Lu-PSMA Administration}

All procedures were performed by the femoral route under local anesthesia (prilocaine hydrochloride $1 \%$, $10 \mathrm{cc}$ ) without sedation with fluoroscopic guidance by using a monoplane angiographic system (Allura Xper FD20/10 Philips Medical Systems, the Netherlands). Pelvic angiography was performed using a 4-F pigtail catheter (TEMPO AQUA®, Cordis, Miami, FL) placed in the distal abdominal aorta. After roadmaps were obtained, the origin of the right internal iliac artery was selectively catheterized using a 4-F modified Simmons type- 1 catheter (Boston Scientific, Marlborough, MA) and the left internal iliac artery was selectively catheterized with a 4-F modified Simmons type-1 or cobra catheter (TEMPO AQUA ${ }^{\circledR}$, Cordis, Miami, FL). The second treatment of the ${ }^{177}$ Lu-PSMA (second half of the total dose $=100 \mathrm{mCi}$ ) was given in the left and right internal iliac arteries successively after angiography (Supplement 1 and 2).

\section{Whole-Body and SPECT/CT Imaging}

One advantage of Lu-177 is that it emits gamma rays on decay, which enables image acquisition and dosimetric calculation using single-photon emission computed tomography (SPECT) or planar scintigraphy. We acquired planar whole-body and SPECT/CT (Siemens Medical Solutions, Erlangen, Germany) scans of the prostate and/or metastatic lesion sites with 3/8-inch crystal thickness using medium energy parallel hole collimators to calculate the absorbed doses, after both IV and IA administrations of ${ }^{177}$ Lu-PSMA. An additional acquisition was made just before IA administration in order 
to obtain a baseline image for background correction. Regions of interest (ROIs) were drawn over the composite image of all axial SPECT slices with increased tracer uptake, with anatomic correlation of CT.

\section{Statistical Analysis}

Absorbed doses in all metastatic lesions, kidneys, liver, bone marrow, prostate, and whole body were determined for each patient using OLINDA/EXM 1.0 software (Vanderbilt University, CTTC, 2004, Nashville, TN) according to the Medical Internal Radiation Dose (MIRD) schema. Mean absorbed dose values of the dominant lesion (i.e. the metastatic lesion with the highest radioactivity count), all lesions, kidneys, liver, bone marrow, prostate, and whole body were calculated and compared between both administration routes. In addition, the absorbed dose value of the dominant lesion and the mean absorbed dose value across all lesions were used to calculate average lesion-to-liver, lesion-to-bone marrow, and lesion-to-whole body ratios, which were also compared between both administration routes. All comparisons were made using Student's $t$-test and $p$-values less than 0.05 were accepted as significant.

\section{Results}

Four patients with mCRPC were included in this study (median age, 62.5 years; range, 53-72). Median PSA values were 89.75 (range, 11.74-173).

Tables 1 and 2 show the absorbed dose values in metastatic lesions, kidneys, liver, bone marrow, prostate, and whole body for each patient after $I V$ and $I A$ administration of ${ }^{177}$ Lu-PSMA, respectively. Table 3 shows the mean absorbed dose value in the dominant lesion (the metastatic lesion with highest count), all lesions, kidneys, liver, bone marrow, prostate, and whole body. Although dominant lesions had a higher mean absorbed dose by $I A$ vs $I V$ administration (2.00 vs $1.35 \mathrm{MGy} / \mathrm{MBq}$ ), the difference was not significant $(\mathrm{p}=0.098)$. By contrast, the mean total absorbed dose of all lesions was significantly higher by $I A$ vs $I V$ administration (1.59 vs $1.20 \mathrm{MGy} / \mathrm{MBq} ; \mathrm{p}=0.024$ ). The prostate gland as a whole had a lower mean absorbed dose for $I A$ vs $I V$ administration ( 0.27 vs $0.36 \mathrm{MGy} / \mathrm{MBq}$ ) but this difference was not significant $(\mathrm{p}=0.22)$. By contrast, the liver mean absorbed dose was significantly lower for the $I A$ vs $I V$ administration ( 0.11 vs $0.13 ; \mathrm{p}=0.04)$. Whole-body dosimetry was in favor of $I A$ vs $I V$ administration but not statistically significant ( 0.05 vs $0.07 ; p=0.18$ ). When lesion-to-liver, lesion-to-bone marrow, lesion-toprostate, and lesion-to-whole body ratios were compared between administration routes, the differences between $I A$ and $I V$ administration were significant $(\mathrm{p}=0.039$ when only the dominant lesions were used to calculate ratios, and $p=0.041$ when all lesions were used to calculate ratios).

All numbers displayed in Table 3 are also illustrated in graphs showing that $I A$ administration was favorable to $I V$ administration (Figs. 2 and 3 ).

Patients tolerated the $I A$ administration using femoral access very well and after control of bleeding, patients left the catherization laboratory without complications, after an average length-of-stay of $3.2 \pm 1.2$ hours following percutaneous intervention. There were no unexpected side effects during the early posttherapy period in all patients and after a one-night stay, patients were discharged safely. None of the four patients experienced more than regularly expected side effects within the first 8-16 weeks of follow-up. 
Several grade 1 adverse effects (xerostomia, nausea, and fatigue) were reported. While early reports of ${ }^{177}$ Lu-PSMA showed a grade 3 or 4 hematological toxicity rate of up to $10 \%$, there were no reports of bleeding or febrile neutropenia in our patients and no acute renal toxicity.

\section{Discussion}

An alternative to the traditional $I V$ administration of chemotherapeutic agents is $I A$ administration, the use of which is expanding. In $I A$ administration, arterial access is first obtained and then fluoroscopic guidance is used to achieve super-selective catheter placement within the arterial supply of a tumor. The goal of $I A$ is to increase local (and subsequently intra-tumoral) concentration of chemotherapeutic agents, decrease systemic adverse effects, and potentially generate greater tumor response [8]. In this preliminary study in a small group of patients with mCRPC, we performed $I A$ and $I V$ administration of ${ }^{177}$ Lu-PSMA. We found that $I A$ administration had favorable results to $I V$ administration in terms of absorbed doses in metastatic lesions without compromising the relevant therapeutic effects.

Intra-arterial administration has been studied to deliver chemotherapeutic agents. In a non-randomized crossover-like diagnostic imaging study by Haberkorn et al, Ga-68 DOTATOC PET/CT was performed after both $I V$ and $I A$ administration; the standardized uptake values of DOTATOC in neuroendocrine tumors were approximately 3.75 times higher after $I A$ administration [9]. An ongoing study in patients with neuroendocrine tumors, the LUTIA (Lutetium Intra-Arterial) study, aims to increase the tumor-absorbed dose in liver metastases $I A$ vs $I V$ administration of ${ }^{177}$ Lu-Dotate [10]. This study will compare ${ }^{177}$ Lu-Dotate activity in tumors in the $I A$-treated lobe with that in the $I V$-treated contralateral lobe, whereby the investigators will specifically evaluate whether there is a difference in tumor-to-non-tumor activity concentration ratio on post-treatment SPECT/CT between $I A$ - and $I V$-treated liver lobes.

${ }^{177}$ Lu-PSMA is a promising therapy for patients with MCRPC [11]. To our knowledge, this is the first study to compare the pharmacokinetic profiles of ${ }^{177}$ Lu-PSMA therapy delivered IA vs $I V$ in patients with mCRPC. The majority of guidelines recommend at least four consecutive treatment cycles of radioligand therapy with 2 months intervals for patients with MCRPC. Since it is challenging to determine the superiority of one administration route over another under these circumstances, we conducted a study using a within-patient comparison design whereby we aimed to calculate the absolute uptake of the given therapeutic dose by $I A$ administration in comparison to the commonly performed $I V$ route, dividing the total empirical therapeutic dose between the two administrations.

In our limited study population of four patients, we found that the absorbed dose to the prostate was similar between both administrations. The possible reason for this could be the high activity crossover from the urinary bladder and the low resolution of ${ }^{177}$ Lu-PSMA whole body SPECT/CT images to show the dominant intraprostatic lesion, so that an exact dosimetric comparison for the dominant tumor is not possible. Using positron emission tomography/magnetic resonance imaging (PET/MRI) images with higher resolution and additional diagnostic parameters such as diffusion-weighted imaging (DWI) and apparent diffusion coefficient (ADC) maps for region of interest selection (ROI) would offer more accurate 
absorbed dose calculations for prostate lesions. Nevertheless, our study still showed that, in spite of its invasive nature, $I A$ administration had a lower radiation burden on the liver and more favorable therapeutic effects in distant metastatic lesions.

While $I A$ administration might be favorable to $I V$ administration, there a few barriers to performing $I A$ administration. First, only individuals who have significant experience with interventional procedures and instrumentation should perform $I A$ procedures. Second, the procedure requires anesthesia. Third, IA administration requires radiological contrast media which always poses inherent risks.

One possible application of $I A$ administration might be in the use of radionuclide therapy in combination with prostate artery embolization. Although not explored in this study, methods to increase the temporal exposure of the radiopharmaceutical being infused could result in additional uptake by the tumor. This could be accomplished by temporary balloon occlusion of proximal arteries or vascular embolization while ${ }^{177}$ Lu-PSMA is being administered. It would be interesting to study the therapeutic consequences of such alternative methods in further prospective studies.

This study has a few limitations. As stated above, firstly, the number of patients included in this study is very small. Larger studies are needed to validate our findings. Secondly, one can expect any possible saturation of PSMA receptors as a consequence of two treatments performed one week apart. Thirdly, it was difficult to select an accurate ROI on prostate due to the low spatial resolution of images and for that reason the exact dosimetry towards the primary pathology could not be accurately demonstrated.

\section{Conclusion}

These preliminary results of an ongoing clinical study showed us that the $I A$ administration of ${ }^{177} \mathrm{Lu}$ PSMA in this small group of MCRPC patients was a safe and feasible approach to $I V$ administration. Since absorbed doses in metastatic lesions were higher with $I A$ administration without compromising the relevant therapeutic effects, we are encouraged to propose the interventional route as an alternative to selected patients with consent and designed a prospective randomized study to understand the long-term efficacy of $I A$ administration considering progression free survival or overall survival, etc. in larger cohorts of patients with mCRPC.

\section{Declarations}

\section{ETHICS APPROVAL:}

This study is approved by The Cerrahpasa Medical Faculty ethics committee and retrospectively registered with file no: 83045809-604.01.02.

\section{AVAILABILITY OF DATA AND MATERIALS:}

The datasets used and/or analysed during the current study are available from the corresponding author on reasonable request. 
COMPETING INTERESTS:

The authors declare that they have no competing interests.

\section{AUTHORS CONTRIBUTIONS:}

HBS: Conceptualization, Methodology, data curation and interpretation, writing the manuscript

FG: Methodology, data curation and interpretation

SS: Critical revision

EA: Data acquisition

NY: Formal data analysis

SB: Data acquisition

KNT: Data acquisition

FB: Data acquisition

RO: Critical revision

OA: Writing-editing, reviewing

\section{ACKNOWLEDGEMENTS:}

We acknowledge Ms Susan D. Weil-Kazzaz, Digital Asset and Production Manager in Memorial Sloan Kettering Cancer Center New York for her kind improvements of figure 1.

\section{References}

[1] Ahmed Y, Osman N, Sheikh R, Picardo S, Watson G. Real-world experience with abiraterone in metastatic castration-resistant prostate cancer. Cancer Translational Medicine. 2017;3:133-8.

[2] Ramadan WH, Kabbara WK, Al Basiouni Al Masri HS. Enzalutamide for patients with metastatic castration-resistant prostate cancer. Onco Targets Ther. 2015;8:871-6.

[3] Hussain M, Mateo J, Fizazi K, Saad F, Shore ND, Sandhu S, et al. LBA12_PR - PROfound: Phase III study of olaparib versus enzalutamide or abiraterone for metastatic castration-resistant prostate cancer (mCRPC) with homologous recombination repair (HRR) gene alterations. Annals of Oncology. 2019;30:v881-v2.

[4] Parker C, Nilsson S, Heinrich D, Helle SI, O'Sullivan JM, Fosså SD, et al. Alpha emitter radium-223 and survival in metastatic prostate cancer. N Engl J Med. 2013;369:213-23. 
[5] Kantoff PW, Higano CS, Shore ND, Berger ER, Small EJ, Penson DF, et al. Sipuleucel-T immunotherapy for castration-resistant prostate cancer. N Engl J Med. 2010;363:411-22.

[6] Pomper MG, Musachio JL, Zhang J, Scheffel U, Zhou Y, Hilton J, et al. 11C-MCG: synthesis, uptake selectivity, and primate PET of a probe for glutamate carboxypeptidase II (NAALADase). Mol Imaging. 2002;1:96-101.

[7] US Department of Health and Human Services. Common Terminology Criteria for Adverse Events (CTCAE) version 5.0. 2017.

[8] von Scheel J, Golde G. Pharmacokinetics of intra-arterial tumour therapy. An experimental study. Arch Otorhinolaryngol. 1984;239:153-61.

[9] Kratochwil C, Giesel FL, López-Benítez R, Schimpfky N, Kunze K, Eisenhut M, et al. Intraindividual comparison of selective arterial versus venous 68Ga-DOTATOC PET/CT in patients with gastroenteropancreatic neuroendocrine tumors. Clin Cancer Res. 2010;16:2899-905.

[10] Ebbers SC, Braat A, Moelker A, Stokkel MPM, Lam M, Barentsz MW. Intra-arterial versus standard intravenous administration of lutetium-177-DOTA-octreotate in patients with NET liver metastases: study protocol for a multicenter, randomized controlled trial (LUTIA trial). Trials. 2020;21:141.

[11] van Kalmthout LWM, van der Sar ECA, Braat AJAT, de Keizer B, Lam MGEH. Lutetium-177-PSMA therapy for prostate cancer patients-a brief overview of the literature. Tijdschrift voor Urologie. 2020;10:141-6.

\section{Tables}

\begin{tabular}{|lllllllllll|}
\hline \multicolumn{7}{|l|}{ Table 1 Absorbed Dose (mGy/MBq) by Intravenous Application } \\
\hline Patient & $\begin{array}{l}\text { Lesion } \\
\mathbf{1}\end{array}$ & $\begin{array}{l}\text { Lesion } \\
\mathbf{2}\end{array}$ & $\begin{array}{l}\text { Lesion } \\
\mathbf{3}\end{array}$ & $\begin{array}{l}\text { Lesion } \\
\mathbf{4}\end{array}$ & Kidneys & Liver & $\begin{array}{l}\text { Bone } \\
\text { marrow }\end{array}$ & Prostate & $\begin{array}{l}\text { Whole } \\
\text { body }\end{array}$ \\
\hline 1 & 1.54 & 1.33866 & 1.35 & 0.134 & 0.214 & 0.121 & 0.0235 & 0.246 & 0.0324 \\
\hline 2 & & & & & 0.89 & 0.19 & 0.13 & 0.27 & 0.18 \\
\hline 3 & 0.32 & 0.35 & 0.7 & & 0.21 & 0.08 & 0.03 & 0.55 & 0.04 \\
\hline 4 & 2.75 & 2.35 & & & 0.34 & 0.14 & 0.03 & & 0.04 \\
\hline
\end{tabular}




\begin{tabular}{|c|c|c|c|c|c|c|c|c|c|}
\hline Patient & $\begin{array}{l}\text { Lesion } \\
1\end{array}$ & $\begin{array}{l}\text { Lesion } \\
2\end{array}$ & $\begin{array}{l}\text { Lesion } \\
3\end{array}$ & $\begin{array}{l}\text { Lesion } \\
\mathbf{4}\end{array}$ & Kidneys & Liver & $\begin{array}{l}\text { Bone } \\
\text { marrow }\end{array}$ & Prostate & $\begin{array}{l}\text { Whole } \\
\text { body }\end{array}$ \\
\hline 1 & 2.51 & 1.646 & 2.58 & 0.214 & 0.195 & 0.114 & 0.0198 & 0.302 & 0.0275 \\
\hline 2 & & & & & 0.576 & 0.143 & 0.066 & 0.209 & 0.092 \\
\hline 3 & 0.28 & 0.4 & 0.46 & & 0.13 & 0.05 & 0.03 & 0.3 & 0.04 \\
\hline 4 & 3.16 & 3.02 & & & 0.36 & 0.13 & 0.03 & & 0.04 \\
\hline
\end{tabular}

\begin{tabular}{|llllll|}
\hline \multicolumn{5}{|l}{ Table 3 Average Absorbed Doses (MGy/MBq) } \\
\hline Application Type & Kidney & Liver & Bone marrow & Prostate & Whole body \\
\hline IV & 0.41 & 0.13 & 0.05 & 0.36 & 0.07 \\
\hline IA & 0.32 & 0.11 & 0.04 & 0.27 & 0.05 \\
\hline P-value & 0.14 & 0.04 & 0.18 & 0.22 & 0.18 \\
\hline & DL & DL/liver & DL/bone marrow & DL/prostate & DL/whole body \\
\hline IV & 1.35 & 10.21 & 24.94 & 3.80 & 18.47 \\
\hline IA & 2.00 & 18.31 & 54.87 & 7.40 & 40.10 \\
\hline P-value & 0.098 & 0.039 & & & \\
\hline & AL & AL/liver & AL/bone marrow & AL/prostate & AL/whole body \\
\hline IV & 1.20 & 9.10 & 22.24 & 3.39 & 16.47 \\
\hline IA & 1.59 & 14.51 & 43.50 & 5.87 & 31.79 \\
\hline P-value & 0.024 & 0.041 & & & \\
\hline
\end{tabular}

Abbreviations: $\mathrm{DL}$, dominant lesion; $\mathrm{AL}$, all lesions

\section{Figures}




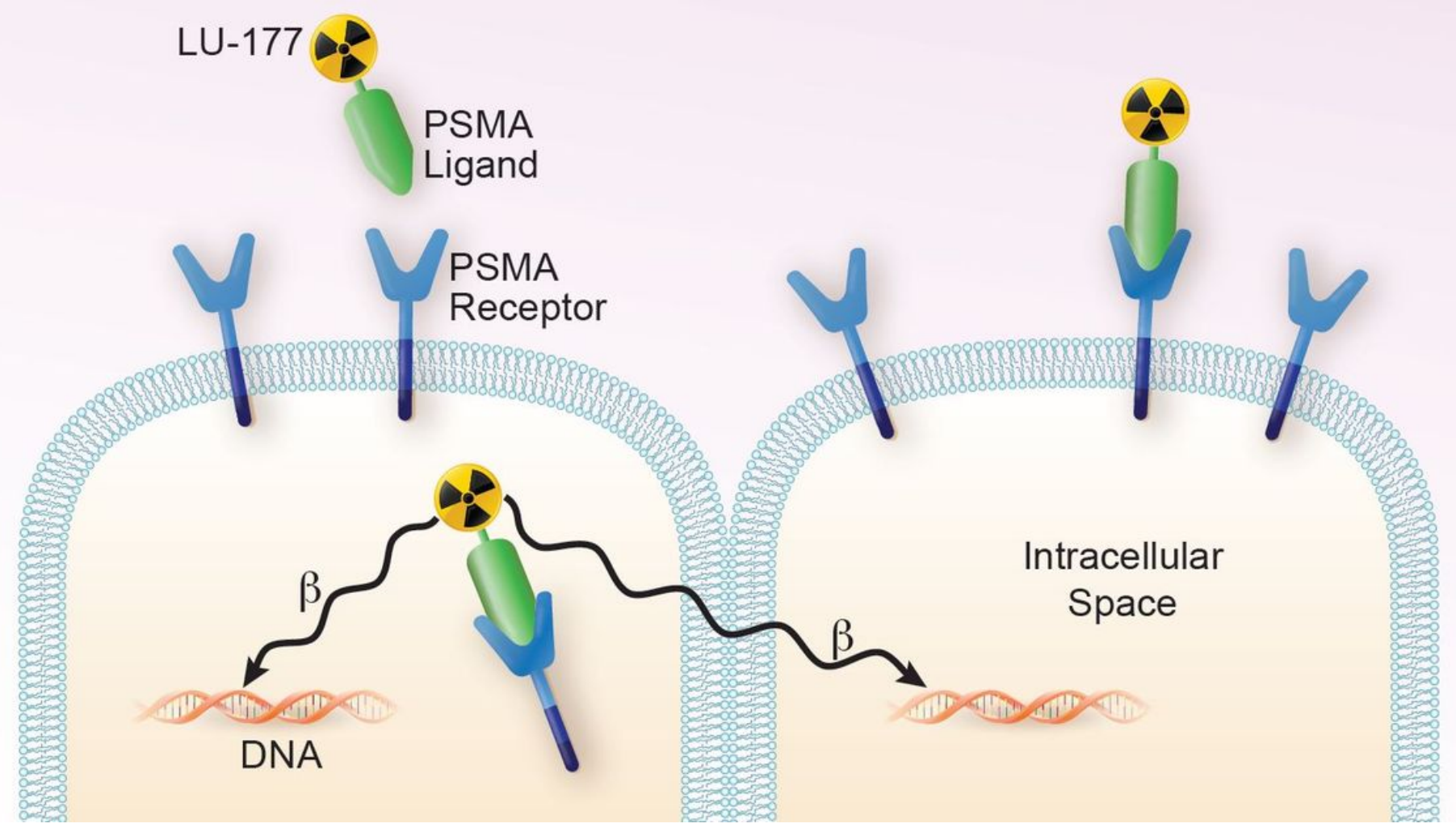

\section{Figure 1}

These small molecules complexed with Lu-177 penetrate solid tumors and have the advantage of rapid clearance from the blood as compared with whole antibodies, which enables the delivery of high doses of cell destructive beta radiation to tumors whiles limiting adjacent bone marrow irradiation. Lu-177 labeled PSMA radiopharmaceuticals (177Lu-PSMA) administered intravenously have been shown to bind rapidly to PSMA receptors and become internalized in cells whereby they remain with a half-life of 6.7 days and have a maximal tissue penetration of approximately $2 \mathrm{~mm}$, exerting a crossfire effect 


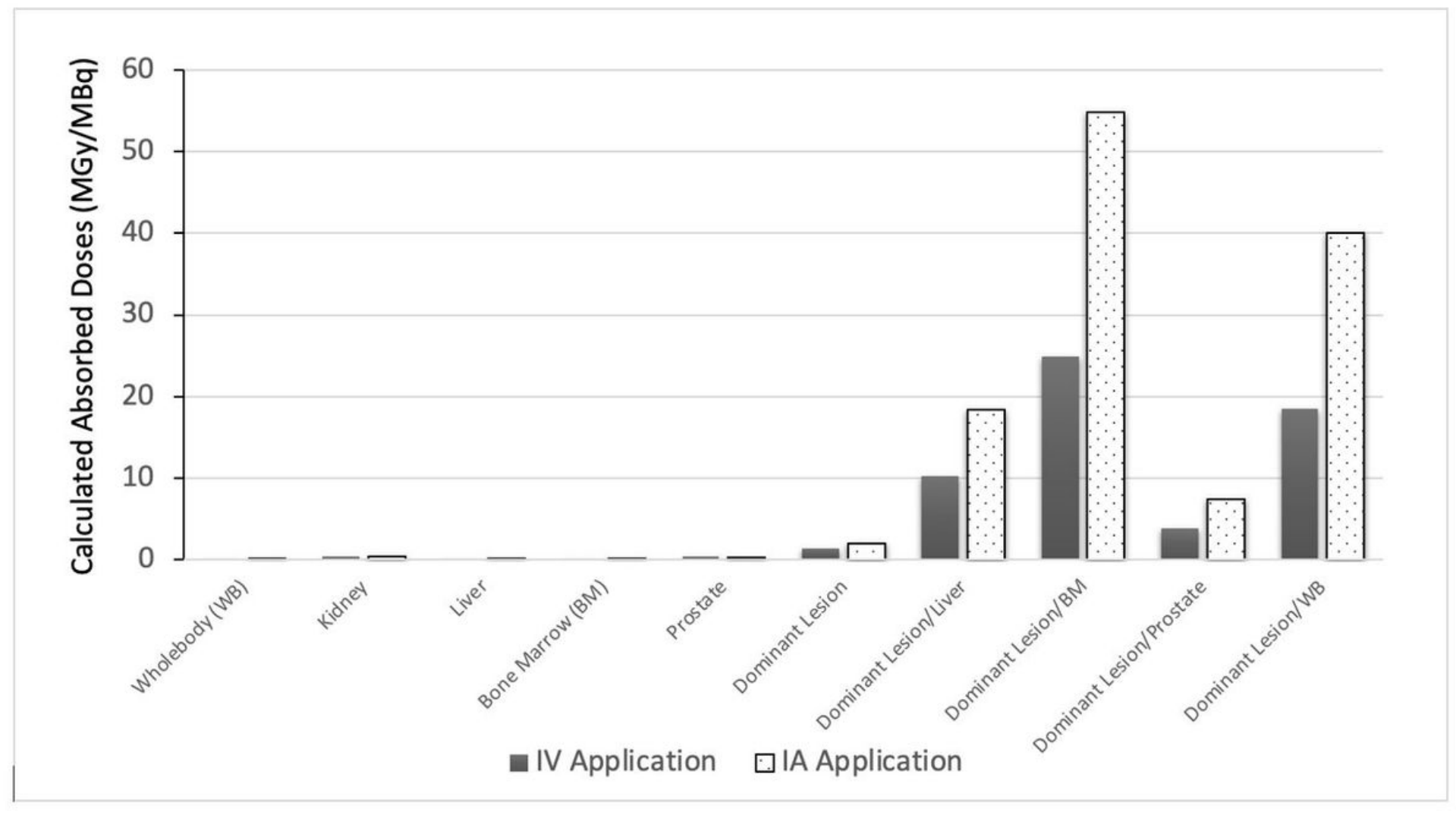

Figure 2

The differences between IA and IV administration were significant $(p=0.039$ when only the dominant lesions were used to calculate ratios, and $p=0.041$ when all lesions were used to calculate ratios).

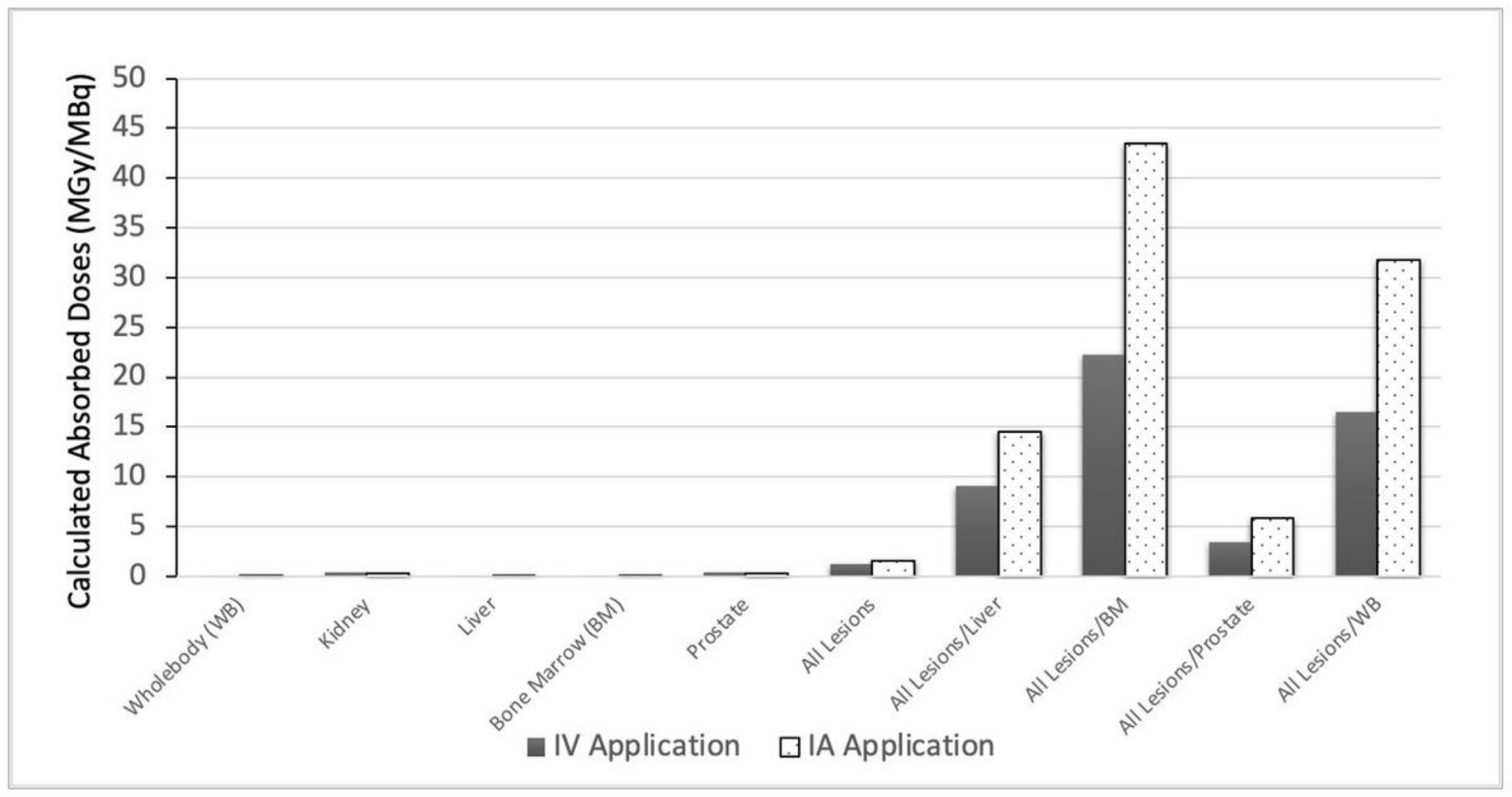

Figure 3 
Patients tolerated the IA administration using femoral access very well and after control of bleeding, patients left the catherization laboratory without complications, after an average length-of-stay of $3.2 \pm$ 1.2 hours following percutaneous intervention

\section{Supplementary Files}

This is a list of supplementary files associated with this preprint. Click to download.

- Supplement1.mov

- Supplement2.mov 